Revisión narrativa o de literatura

\title{
INTERVENCIONES NO FARMACOLÓGICAS REALIZADAS POR ENFERMERÍA PARA PROMOVER LA CESACIÓN DEL TABACO
}

\author{
NON-PHARMACOLOGICAL INTERVENTIONS PERFORMED BY NURSING TO \\ PROMOTE TOBACCO CESSATION
}

\section{Claudia Liliana Valencia Rico}

Enfermera. Especialista en Rehabilitación Cardiopulmonar. Magister en Enfermería con énfasis en cuidado al adulto y anciano. Profesora Programa Enfermería. Universidad Católica de Manizales. Carrera 23 \# 60- 63, Caldas. Colombia.

\section{Valentina Palacio Noreña}

Estudiante Programa Enfermería. Universidad Católica de Manizales. Carrera 23 \# 60- 63, Caldas. Colombia.

\section{Laura Vanessa Villada Pineda}

Estudiante Programa Enfermería. Universidad Católica de Manizales. Carrera 23 \# 60- 63, Caldas. Colombia.

\section{Katherin Giraldo Castaño}

Estudiantes Programa Enfermería. Universidad Católica de Manizales. Carrera 23 \# 60- 63, Caldas. Colombia.

Artículo recibido el 10 de julio de 2018. Aceptado en versión corregida el 13 de diciembre de 2018.

\section{RESUMEN}

Objetivo: describir las intervenciones no farmacológicas realizadas por enfermería que han sido reportadas en la literatura actual para promover el cese del consumo de tabaco. Método: revisión integradora de literatura mediante búsqueda en bases de datos Google Académico, Scopusc, Cochrane y Pubmed. Se tomaron los artículos publicados entre los años 2014 a 2018. Los principales descriptores de búsqueda fueron: "tobacco use cessation" y "nursing care". Se incluyeron un total de 11 manuscritos que fueron analizados por nivel de evidencia, tipo de intervención y principales resultados. Resultados: el 54,5\% de los manuscritos correspondía a publicaciones de evidencia media, 36,4\% estudios de evidencia alta y el 9,1\% evidencia baja, según criterios de Jover y Navarro-Rubio. Las intervenciones mayormente realizadas por enfermería se relacionan con la asesoría breve e intensiva cara a cara y el asesoramiento telefónico. Conclusión: la mayor parte de los estudios analizados se ubican en evidencia media y alta. Enfermería logra demostrar con sus intervenciones el aumento de las tasas de abstinencia puntual en todos los estudios; a pesar de esto, la evidencia muestra 
que se requiere de una mayor preparación desde el proceso enfermero para poder intervenir y promover el cese del tabaco.

Palabras clave: Cese del uso de tabaco, Cuidado de Enfermería, Consejería (Fuente DECS BIREME).

\begin{abstract}
Objective: to describe the non-pharmacological interventions carried out by nursing that have been reported in the current literature to promote cessation of tobacco use. Method: integrative review of literature through Google Scholar, Scopusc, Cochrane and Pubmed databases. The articles published between 2014 and 2018 were taken. The main search descriptors were: "tobacco use cessation" and "nursing care". A total of 11 manuscripts were included that were analyzed by level of evidence, type of intervention and main results. Results: $54.5 \%$ of the manuscripts corresponded to publications of medium evidence, $36.4 \%$ studies of high evidence and $9.1 \%$ low evidence, according to criteria of Jover and NavarroRubio. Interventions mainly carried out by nursing are related to brief and intensive face-toface counseling and telephone counseling. Conclusion: most of the studies analyzed are located in medium and high evidence. Nursing is able to demonstrate with its interventions the increase of the rates of punctual abstinence in all the studies; In spite of this, the evidence shows that a greater preparation is required from the nursing process in order to intervene and promote tobacco cessation.
\end{abstract}

Keywords: Tobacco Use Cessation, Nursing Care, Counseling.

\title{
http://dx.doi.org/10.7764/Horiz_Enferm.29.3.212-223
}

\section{INTRODUCCIÓN}

En la actualidad el consumo de tabaco se considera un problema de salud pública; las estadísticas epidemiológicas mundiales con relación al tabaquismo muestran que este tipo de adicción mata hasta a la mitad de sus consumidores produciendo más de 6 millones de muertes por año, de las cuales, un promedio de 890.000 son fumadores pasivos. Adicionalmente, de los mil millones de fumadores que hay en el mundo, el $80 \%$ viven en países de ingresos bajos o medios $^{1}$. En consecuencia, el consumo de tabaco se ha considerado como uno de los hábitos más utilizados y diseminados en la sociedad contemporánea; su uso es la principal causa de muerte prevenible entre hombres y mujeres, evidenciándose una tendencia al crecimiento de este hábito en las mujeres ${ }^{2}$.

En Colombia se ha notificado que 21.765 personas mueren anualmente por enfermedades atribuibles al consumo del tabaco y que el recaudo anual por sus impuestos es de 400 mil millones de pesos $^{3}$. El país es pionero en Suramérica, en lo que respecta a contribuir con la abolición de la publicidad, promoción y patrocinio del tabaco, ya que se tiene establecida la prohibición total de toda 
Intervenciones no farmacológicas realizadas por...

forma de promover este producto y sus derivados ${ }^{4}$; sin embargo, pese a los esfuerzos realizados en el sector salud por contribuir al cese de este hábito, las estadísticas y los estudios muestran que poblaciones cada vez más jóvenes incursionan en el consumo ${ }^{5-7}$.

Teniendo en cuenta la problemática con relación al consumo de tabaco y su factor de riesgo potencial, sobre todo a nivel cardiopulmonar ${ }^{8}$, desde enfermería se han implementado diversas intervenciones para contribuir a la cesación de este hábito, utilizando tratamientos de tipo no farmacológico o tratamientos conjugados con terapia farmacológica, que han resultado ser más efectivos, ya que bloquean de manera positiva los efectos placenteros de la nicotina $^{9,10}$.

Dentro de las estrategias no farmacológicas utilizadas se pueden mencionar las terapias cognitivoconductuales, que han brindado resultados satisfactorios en la cesación del uso de tabaco y sesiones de consejería rápida e intensiva, permitiendo el abandono continuo del cigarrillo, con ganancias en la calidad de vida de las personas ${ }^{11}$; así mismo, la estrategia de llamadas y mensajes de texto, la promoción de líneas de ayuda mediante campañas de medios masivos como televisión, radio, internet y anuncios impresos; así como la práctica del ejercicio físico y las respiraciones yóguicas, han sido utilizadas en diversos estudios intentando demostrar su eficacia sobre la abstinencia puntual ${ }^{12,13}$.

Por otro lado, se puede mencionar la utilización de cigarrillo electrónico (CE), al que se le han atribuido tres posibles categorías de uso: como producto medicinal, como producto de tabaco o como producto de consumo; sin embargo, los estudios realizados con este método para el cese del consumo de tabaco no han demostrado hallazgos significativos en comparación con otros métodos o intervenciones, por tal motivo, se ha recomendado que la utilización del CE sea regulada como una medicación, dado el uso indiscriminado del mismo, que podría traer igualmente repercusiones para la salud $^{14}$.

Para enfermería es un reto poder realizar intervenciones efectivas con las que pueda conseguir el logro de la abstinencia puntual y/o continua en los grupos de personas que son atendidos en todos los contextos del cuidado ${ }^{15}$. En este sentido, el trabajo interdisciplinar es necesario para lograr resultados significativos y potenciar dentro de cada uno de los roles profesionales un mejor acompañamiento a la persona fumadora; para esto, los profesionales de enfermería deben estar capacitados en lo que respecta a las técnicas del cese del tabaco ${ }^{16,17}$.

En el presente artículo se analizaron las publicaciones científicas actuales realizadas por enfermería para promover el cese del consumo de tabaco en personas fumadoras mediante intervenciones no farmacológicas.

\section{MÉTODO}

Revisión integradora que se llevó a cabo mediante las siguientes fases: delimitación de la pregunta orientadora y el objetivo, identificación de descriptores, determinación de los criterios de inclusión, búsqueda sistemática en bases de datos, análisis de los estudios encontrados y determinación de las principales 
Valencia, C., Palacio, V., Villada, L., Giraldo, K.

intervenciones con sus resultados. La pregunta orientadora que dio origen a la búsqueda fue la siguiente: ¿Qué tipo de intervenciones no farmacológicas han sido realizadas por los profesionales de enfermería para promover la cesación del consumo de tabaco?

Se realizó una búsqueda de artículos en las siguientes bases bibliográficas: Google Académico, Scopus, Cochrane y Pubmed. Los términos utilizados para la búsqueda a través del Decs Bireme fueron los siguientes: tobacco use cessation, nursing care, education nursing.

La selección inicial de los artículos se realizó por título obteniendo un total de 66 artículos. Como criterios de inclusión se precisaron, artículos originales derivados de investigaciones de cualquier tipo y alcance, metaanálisis y revisiones sistemáticas de literatura publicados a partir del año 2014. Se excluyeron los artículos de revisión de tema, artículos cortos y artículos de reflexión no derivados de investigación, de esta forma se obtuvo una muestra definitiva de 11 artículos (Figura 1).

Para el análisis de los manuscritos seleccionados se tuvo en cuenta: el nivel de evidencia aportado, según criterios de Jover y Navarro-Rubio ${ }^{18}$, el tipo de intervención realizada y sus principales resultados.

\section{RESULTADOS}

El análisis de los 11 artículos seleccionados muestra que, el 54,5\% de las publicaciones correspondían a estudios de evidencia media (cuatro estudios cuasiexperimentales y dos revisiones sistemáticas de literatura); el $36,4 \%$ 2018, Horiz. Enferm., 28,3,212-223 correspondía a estudios de evidencia alta (cuatro ensayos clínicos con asignación aleatoria) y el $9,1 \%$ restante, trataba de estudios de evidencia baja (un estudio descriptivo) (Tabla 1).

En cuanto a las intervenciones encontradas se puede decir que la mayor parte de ellas corresponden estrategias de consejería breve, consejería intensiva y asesoría telefónica. Dichas estrategias se han utilizado mayoritariamente de manera combinada con otras como: la entrega adicional de folletos informativos, visitas de seguimiento presencial y utilización de mensajes de texto para el seguimiento y control de las variables de interés en la cesación del tabaco. Otras técnicas menos utilizadas fueron las terapias cognitivo conductuales y la utilización de la técnica mindfulness (Tabla 1).

Un aspecto importante de resaltar es que, en varios de los estudios encontrados, se realizó entrenamiento previo a los profesionales de enfermería sobre las tácticas en cesación de tabaco, tanto en contextos clínicos como hospitalarios; en este sentido, se reporta la enseñanza de estrategias para la captación de personas fumadoras y su abordaje inicial basado en la técnica de las 5 As (preguntar sobre el consumo de tabaco, aconsejar dejar de fumar, evaluar la disposición del sujeto a renunciar, ayudar en el intento de dejar de fumar, y organizar el seguimiento). En este sentido, los estudios se encargaron de medir no solo las variables relacionadas con el cese del tabaquismo en las personas intervenidas, sino que también precisaron la adopción y adherencia de las estrategias antitabaco por parte de los profesionales que fueron entrenados para realizarlas. 
Por otro lado, en cuanto a los resultados encontrados, se puede decir que en todos los estudios se tuvo como resultado primario la abstinencia puntual (generalmente a los 7 días, 1,3, 6 y 12 meses de la intervención) y otras variables de interés que eran específicas de las diversas poblaciones atendidas. Si bien todas las intervenciones demuestran haber logrado aumentar las tasas de abstinencia, no se logra identificar cual de todas las estrategias utilizadas pudo llegar a ser más intensiva con visitas de seguimiento presencial, se mostraron mejores resultados, así mismo sucedió con los métodos impersonales, donde se realizaron seguimientos telefónicos. También se puede mencionar que solo un estudio utilizó intervención farmacológica conjugada con la consejería realizada por enfermería, demostrando resultados significativos en la abstinencia.

Figura 1. Descripción de la búsqueda y elección de los manuscritos. Fuente: elaboración propia.
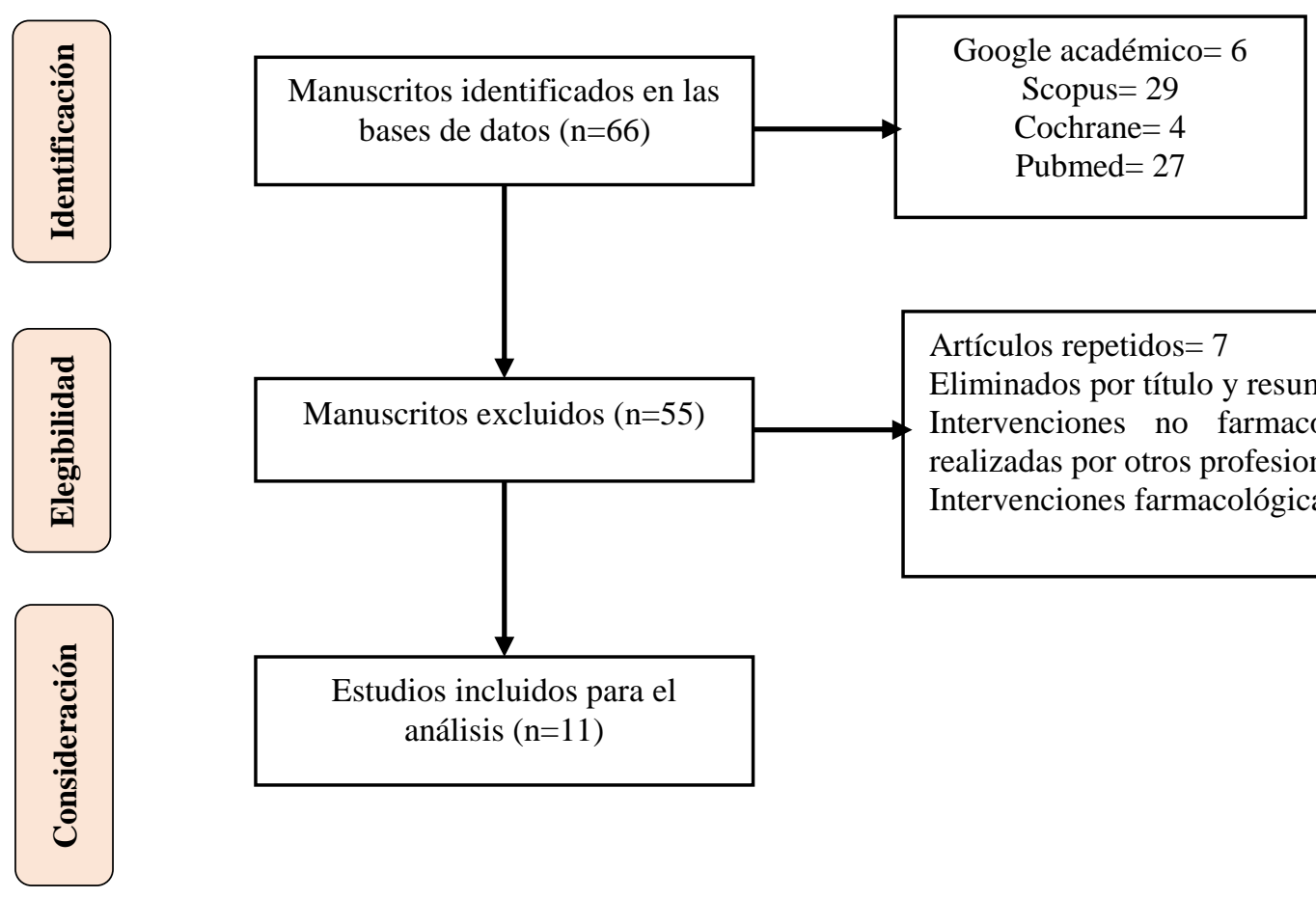

Estudios incluidos para el análisis $(\mathrm{n}=11)$

Artículos repetidos $=7$

Eliminados por título y resumen=39

Intervenciones no farmacológicas

realizadas por otros profesionales $=4$

Intervenciones farmacológicas $=5$ 


\section{Intervenciones no farmacológicas realizadas por...}

Tabla 1. Descripción de las intervenciones encontradas.

\begin{tabular}{|c|c|c|c|}
\hline Publicación & Tipo Estudio & Intervención(es) realizada & Principales resultados \\
\hline $\begin{array}{l}\text { Economic } \\
\text { evaluation of a } \\
\text { telephone- and } \\
\text { face-to-face- } \\
\text { delivered } \\
\text { counseling } \\
\text { intervention for } \\
\text { smoking cessation } \\
\text { in patients } \\
\text { with coronary heart }^{\text {disease }}{ }^{19} \text {. }\end{array}$ & $\begin{array}{l}\text { Ensayo clínico } \\
\text { con asignación } \\
\text { aleatoria }\end{array}$ & $\begin{array}{l}\text { Atención habitual mediante } \\
\text { consejo breve y folleto. } \\
\text { Acompañamiento telefónico: } 7 \\
\text { llamadas telefónicas por } 3 \\
\text { meses. Duración 10-15 min más } \\
\text { TRN. } \\
\text { Consejería cara a cara: } 6 \\
\text { sesiones por } 3 \text { meses. Duración } \\
\text { 40-45min más TRN. }\end{array}$ & $\begin{array}{l}\text { El acompañamiento telefónico más la } \\
\text { adición de terapia de reemplazo con } \\
\text { nicotina, demostró ser la intervención } \\
\text { más rentable Vrs consejería cara a } \\
\text { cara y/o atención habitual. Demostró } \\
\text { una mayor probabilidad de } \\
\text { abstinencia con menores costos } \\
\text { sociales durante } 6 \text { meses. } \\
\text { No hubo diferencias en los años de } \\
\text { vida ajustados por calidad de vida } \\
\text { entre las intervenciones. }\end{array}$ \\
\hline $\begin{array}{l}\text { Brief intervention } \\
\text { to promote smoking } \\
\text { cessation and } \\
\text { improve glycemic } \\
\text { control in smokers } \\
\text { with type } 2 \\
\text { diabetes: a } \\
\text { randomized } \\
\text { controlled trial }{ }^{20} \text {. }\end{array}$ & $\begin{array}{c}\text { Ensayo Clínico } \\
\text { aleatorizado }\end{array}$ & $\begin{array}{l}\text { Intervención breve basada en } \\
\text { asesoramiento individualizada } \\
\text { cara a cara de } 20 \text { minutos por } \\
\text { parte de enfermeras consejeras, } \\
\text { basado en las cinco As. Entrega } \\
\text { de folleto que resumía las } \\
\text { relaciones entre el tabaquismo y } \\
\text { las complicaciones diabéticas. } \\
\text { Visitas de seguimiento de } 1 \\
\text { semana y } 1 \text { mes, con duración } \\
\text { de } 30 \text { minutos para ofrecer un } \\
\text { refuerzo de la intervención } \\
\text { breve destinada a mejorar su } \\
\text { autoeficacia y superar las } \\
\text { barreras para dejar de fumar. } \\
\text { Grupo control: atención } \\
\text { habitual más folleto y consejos } \\
\text { breves. }\end{array}$ & $\begin{array}{l}\text { No se observaron diferencias } \\
\text { significativas en la prevalencia } \\
\text { puntual de } 7 \text { días de la abstinencia de } \\
\text { fumar, el nivel de hemoglobina } \\
\text { glicosilada (HbA1c), la tasa de } \\
\text { abandono validada bioquímicamente, } \\
\text { la tasa de reducción de tabaquismo en } \\
\text { al menos un 50\%, la etapa de } \\
\text { preparación para dejar de fumar o el } \\
\text { número de intentos para dejar de } \\
\text { fumar entre los grupos de intervención } \\
\text { y control a los } 12 \text { meses. } \\
\text { No hubo diferencias significativas en } \\
\text { el nivel de HbA1c entre los que } \\
\text { dejaron de fumar de manera puntual, } \\
\text { pero hubo una tendencia significativa } \\
\text { en la disminución de los que dejaron } \\
\text { de fumar en comparación con la línea } \\
\text { de base después de ajustar algunas } \\
\text { variables. }\end{array}$ \\
\hline $\begin{array}{l}\text { A block } \\
\text { randomized } \\
\text { controlled trial of a } \\
\text { brief smoking } \\
\text { cessation } \\
\text { counselling and } \\
\text { advice through } \\
\text { shortmessage } \\
\text { service on } \\
\text { participants who } \\
\text { joined the Quit to } \\
\text { Win } \\
\text { Contest in Hong } \\
\text { Kong }\end{array}$ & $\begin{array}{l}\text { Ensayo clínico } \\
\text { con asignación } \\
\text { aleatoria }\end{array}$ & $\begin{array}{l}\text { Se ingresaron en un concurso: } \\
\text { un grupo de personas que } \\
\text { recibió } 5 \text { minutos de } \\
\text { asesoramientor telefónico } \\
\text { dirigido por una enfermera; otro } \\
\text { grupo recibió ocho mensajes de } \\
\text { texto a través del teléfono móvil } \\
\text { y un grupo control no recibió } \\
\text { ninguna intervención. }\end{array}$ & $\begin{array}{l}\text { No hubo una diferencia significativa } \\
\text { en la tasa de abandono obtenida con la } \\
\text { asesoría breve telefónica y los } \\
\text { mensajes de texto. Sin embargo, con } \\
\text { el análisis de regresión logística se } \\
\text { logró demostrar que la asesoría breve } \\
\text { telefónica aumentó la abstinencia en } \\
\text { un } 38 \% \text { en los tres seguimientos } \\
\text { realizados. }\end{array}$ \\
\hline $\begin{array}{l}\text { Web-Enhanced } \\
\text { Tobacco Tactics } \\
\text { With Telephone } \\
\text { Support Versus } \\
\text { 1-800-QUIT-NOW } \\
\text { Telephone Line } \\
\end{array}$ & $\begin{array}{c}\text { Ensayo clínico } \\
\text { con asignación } \\
\text { aleatoria }\end{array}$ & $\begin{array}{l}\text { Se evaluó la eficacia y el uso de } \\
\text { las tácticas de tabaco mejoradas } \\
\text { en la Web Vrs la estrategia de } \\
\text { una línea telefónica de ayuda } \\
\text { para dejar de fumar. }\end{array}$ & $\begin{array}{l}\text { El grupo del sitio web de mostró tasas } \\
\text { de abandono del hábito } \\
\text { significativamente más altas que el } \\
\text { grupo de ayuda telefónica a los } 30 \text { días } \\
\text { de seguimiento, pero esta diferencia } \\
\text { ya no fue significativa a los } 6 \text { meses }\end{array}$ \\
\hline
\end{tabular}


Valencia, C., Palacio, V., Villada, L., Giraldo, K.

\begin{tabular}{|c|c|c|c|}
\hline $\begin{array}{l}\text { Intervention for } \\
\text { Operating } \\
\text { Engineers: } \\
\text { Randomized } \\
\text { Controlled Trial }^{22} \text {. }\end{array}$ & & & \\
\hline $\begin{array}{l}\text { A Culturally } \\
\text { Adapted Smoking } \\
\text { Cessation } \\
\text { Intervention for } \\
\text { Korean } \\
\text { Americans }^{23} \text {. }\end{array}$ & $\begin{array}{c}\text { Estudio } \\
\text { cuasiexperimen } \\
\text { tal }\end{array}$ & $\begin{array}{l}\text { Asesoría telefónica más TRN } \\
\text { (parches) por tres meses. Se } \\
\text { incluyó un familiar en el estudio } \\
\text { que verificó la abstinencia de } \\
\text { los participantes. }\end{array}$ & $\begin{array}{l}\text { Las personas mayores percibieron una } \\
\text { norma de pares más fuerte que } \\
\text { favorecía dejar de fuma por este } \\
\text { medio; también demostraron mayor } \\
\text { autoeficacia Vrs los participantes } \\
\text { jóvenes. Los que percibieron una } \\
\text { norma familiar más fuerte para dejar } \\
\text { de fumar tenían una menor } \\
\text { dependencia de la nicotina. La } \\
\text { autoeficacia } \text { se relacionó } \\
\text { inversamente con la dependencia a la } \\
\text { nicotina y con los riesgos percibidos } \\
\text { de dejar de fumar, pero se relacionó } \\
\text { positivamente con la norma familiar } \\
\text { percibida para dejar de fumar. }\end{array}$ \\
\hline $\begin{array}{l}\text { Implementation of } \\
\text { the Tobacco Tactics } \\
\text { intervention versus } \\
\text { usual care in Trinity } \\
\text { Health community } \\
\text { hospitals }^{24} \text {. }\end{array}$ & $\begin{array}{l}\text { Estudio Cuasi- } \\
\text { experimental }\end{array}$ & $\begin{array}{l}\text { Intervención realizada en cinco } \\
\text { hospitales; tres recibieron la } \\
\text { intervención de tácticas de } \\
\text { cesación de tabaco por una } \\
\text { enfermera y dos recibieron la } \\
\text { atención habitual. Se evaluó el } \\
\text { alcance: mediante la tasa de } \\
\text { participación de los pacientes y } \\
\text { su recepción frente al servicio; } \\
\text { la adopción: mediante la tasa de } \\
\text { participación por parte de las } \\
\text { enfermeras y el mantenimiento } \\
\text { de la intervención } \\
\text { implementada en los hospitales. }\end{array}$ & $\begin{array}{l}\text { Alcance: las tasas de participación de } \\
\text { los pacientes fueron del } 71,5 \% \text { con } \\
\text { aumentos significativos posteriores a } \\
\text { la intervención. } \\
\text { Adopción: el } 76.0 \% \text { de las enfermeras } \\
\text { seleccionadas en los hospitales } \\
\text { participaron de la intervención y el } \\
92.4 \% \text { estuvo extremadamente o algo } \\
\text { satisfecho con la capacitación que } \\
\text { recibió. }\end{array}$ \\
\hline $\begin{array}{l}\text { Effectiveness of the } \\
\text { Tobacco Tactics } \\
\text { Program in the } \\
\text { Trinity Health } \\
\text { System }^{25} \text {. }\end{array}$ & $\begin{array}{c}\text { Estudio Cuasi- } \\
\text { expreimnetal }\end{array}$ & $\begin{array}{l}\text { Tres hospitales recibieron la } \\
\text { intervención de tácticas de } \\
\text { tabaco administrada por una } \\
\text { enfermera y tres recibieron la } \\
\text { atención habitual. Los } \\
\text { participantes recibieron } \$ 10 \\
\text { por cada encuesta y } \$ 20 \text { para la } \\
\text { devolución de una prueba de } \\
\text { cotinina. Se realizó seguimiento } \\
\text { telefónico para estimar el estado } \\
\text { de cesación de los participantes } \\
\text { durante } 6 \text { meses. La } \\
\text { intervención incluyó: un folleto, } \\
\text { un DVD de cesación, manual de } \\
\text { tácticas de tabaco, una tarjeta } 1- \\
\text { 800-QUIT-NOW, consejería } \\
\text { conductual y farmacéutica de } \\
\text { enfermería; recordatorios } \\
\text { médicos con consejos breves } \\
\text { para dejar de fumar y } \\
\text { asesoramiento telefónico. }\end{array}$ & $\begin{array}{l}\text { Hubo mejoras significativas en las } \\
\text { tasas de abandono autoinformadas } \\
\text { antes y después de la intervención. Se } \\
\text { evidencian mejores tasas de abandono } \\
\text { verificadas con cotinina y de tasas de } \\
\text { abandono autoinformadas a los } 6 \\
\text { meses en los hospitales de } \\
\text { intervención en comparación con } \\
\text { ningún cambio en los hospitales de } \\
\text { control. }\end{array}$ \\
\hline
\end{tabular}




\section{Intervenciones no farmacológicas realizadas por...}

\begin{tabular}{|c|c|c|c|}
\hline $\begin{array}{l}\text { Intervención breve } \\
\text { multicomponente } \\
\text { de enfermería para } \\
\text { reducir el consumo } \\
\text { de tabaco: un } \\
\text { estudio de } \\
\text { factibilidad }{ }^{26} \text {. }\end{array}$ & $\begin{array}{l}\text { Estudio } \\
\text { cuasiexperimen } \\
\text { tal }\end{array}$ & $\begin{array}{l}\text { Asesoramiento de } 10 \text { semanas. } \\
\text { Sesiones semanales de } 60-90 \\
\text { minutos. Basada en los } \\
\text { programas Mindfulness Based } \\
\text { Stress Reduction y Mindfulness } \\
\text { Based Cognetive Therapy }\end{array}$ & $\begin{array}{l}\text { El número de cigarrillos fumados } \\
\text { diariamente y las puntuaciones en los } \\
\text { instrumentos DASS-21 y Fagerstrom } \\
\text { decrecieron significativamente en el } \\
\text { postest con diferencias } \\
\text { estadísticamente significativas. }\end{array}$ \\
\hline $\begin{array}{l}\text { Nursing } \\
\text { interventions for } \\
\text { smoking } \\
\text { cessation }^{27} \text {. }\end{array}$ & $\begin{array}{c}\text { Revisión } \\
\text { Sistemática de } \\
\text { Literatura }\end{array}$ & $\begin{array}{l}\text { Intervenciones intensidad baja: } \\
\text { ensayos en los que se } \\
\text { proporcionó asesoramiento } \\
\text { (con o sin un folleto) durante } \\
\text { una consulta única que duró } 10 \\
\text { minutos o menos, con hasta una } \\
\text { visita de seguimiento. } \\
\text { Intervenciones intensidad alta: } \\
\text { ensayos en los que el contacto } \\
\text { inicial duró más de } 10 \text { minutos, } \\
\text { hubo materiales adicionales o } \\
\text { estrategias o ambos, aparte de } \\
\text { folletos simples, y } \\
\text { generalmente los participantes } \\
\text { tuvieron más de un contacto de } \\
\text { seguimiento. }\end{array}$ & $\begin{array}{l}\text { La intervención realizada por una } \\
\text { enfermera fue más efectiva que la } \\
\text { atención habitual en la abstinencia de } \\
\text { fumar a los seis meses o más desde el } \\
\text { inicio del tratamiento. La dirección } \\
\text { del efecto fue consistente en diferentes } \\
\text { intensidades de intervención, en } \\
\text { diferentes entornos y en fumadores } \\
\text { con y sin enfermedades relacionadas } \\
\text { con el tabaco. } \\
\text { El apoyo para dejar de fumar por parte } \\
\text { de una enfermera lleva a una modesta } \\
\text { mejora en la abstinencia del tabaco. La } \\
\text { mayoría de estas intervenciones } \\
\text { estuvieron a cargo de enfermeras con } \\
\text { una función especializada en } \\
\text { promoción de la salud. No hubo } \\
\text { pruebas suficientes para saber si las } \\
\text { enfermeras generales pueden lograr } \\
\text { los mismos beneficios. }\end{array}$ \\
\hline $\begin{array}{l}\text { Terapia Cognitivo } \\
\text { Conductual para } \\
\text { Dejar de Fumar: } \\
\text { Revisión } \\
\text { Sistemática }^{28} \text {. }\end{array}$ & $\begin{array}{c}\text { Revisión } \\
\text { Sistemática de } \\
\text { Literatura }\end{array}$ & $\begin{array}{l}\text { Terapia cognitivo-conductual } \\
\text { realizada en } 4 \text { a } 10 \text { sesiones. } \\
\text { Duración } 15 \text { minutos a } 1 \text { hora. }\end{array}$ & $\begin{array}{l}\text { Las terapias cognitivo conductuales } \\
\text { que mostraron mayor efectividad a } \\
\text { corto plazo fueron aquellas en donde } \\
\text { intervención se centró en el desarrollo } \\
\text { de habilidades de afrontamiento para } \\
\text { ayudar a lograr y mantener la } \\
\text { abstinencia del tabaco. Algunos de los } \\
\text { artículos que realizaron un } \\
\text { seguimiento tuvieron una disminución } \\
\text { en las tasas de abstinencia, con } \\
\text { diferencias no significativas en } \\
\text { comparación con el grupo control y } \\
\text { las medidas basales. }\end{array}$ \\
\hline $\begin{array}{l}\text { Efectividad de un } \\
\text { programa de } \\
\text { deshabituación } \\
\text { tabáquica llevado a } \\
\text { cabo en el centro de } \\
\text { salud de } \\
\text { Tapia de Casariego } \\
\text { (Principado de }^{29} \\
\text { Asturias) }\end{array}$ & $\begin{array}{l}\text { Estudio } \\
\text { descriptivo y } \\
\text { retrospectivo. }\end{array}$ & $\begin{array}{l}\text { Intervención multicomponente } \\
\text { realizada a través de técnicas } \\
\text { cognitivas y conductuales } \\
\text { individuales y grupales }(8-12 \\
\text { personas). Cinco sesiones de } 1 \\
\text { hora, con intervalo de una } \\
\text { semana. Se realizó seguimiento } \\
\text { telefónico al año del abandono } \\
\text { del cigarrillo. }\end{array}$ & $\begin{array}{l}\text { Se encontró influencia significativa } \\
\text { entre la puntuación del test de } \\
\text { Richmond y la variable "no fumar al } \\
\text { año de la intervención". Así mismo se } \\
\text { observó asociación entre "haber } \\
\text { recibido intervención grupal" y tener } \\
\text { una mejor puntuación en el test de } \\
\text { Richmond con la variable "no fumar } \\
\text { al año de la intervención". }\end{array}$ \\
\hline
\end{tabular}

Fuente: elaboración propia. 


\section{DISCUSIÓN}

La mayor parte de los estudios encontrados eran de evidencia media y alta; esto sugiere un aporte significativo frente al tema de las intervenciones no farmacológicas realizadas por enfermería para promover el cese del tabaco. A pesar de esto, se debe reconocer que el posicionamiento de enfermería y sus aportes en esta área, es algo que aún necesita ser mayormente trabajado, sobre todo en lo que respecta a los conocimientos, habilidades y destrezas de la población de profesionales de enfermería en general, y no solo de unos pocos profesionales dedicados al tema ${ }^{15-17}$. $\mathrm{Si}$ bien está demostrado que las intervenciones para la cesación del tabaco deben ser trabajadas de manera interdisciplinar, el profesional de enfermería cuenta aún con la posibilidad de desempeñarse en un campo que se encuentra ya bastante explorado, y que sugiere que el enfermero puede posicionar sus modelos de atención en el proceso del cuidado. Al respecto, Olano-Espinosa, et $\mathrm{al}^{30}$, refieren que es necesario realizar intervenciones más sencillas, útiles y basadas en la evidencia que ayuden en el desempeño del trabajo; para ello se requiere que cada profesional sanitario, identifique y aplique las estrategias que puedan ser más efectivas desde su rol. En el caso de enfermería, se ha demostrado que la realización de la consejería breve es una estrategia que poco efectiva en el cese del tabaquismo, en cuyo caso, es mayormente aplicada por profesionales de medicina con demostración de mejores resultados. Caso contrario sucede con la consejería intensiva, cuyo campo es propicio para que enfermería pueda 2018, Horiz. Enferm., 28,3,212-223 aportar significativamente con estrategias educativas, de autoayuda y preparación para el cese del consumo. Adicionalmente, el proceso de acompañamiento durante semanas o meses, es propio del enfermero profesional, quien resulta bastante aportante en la asesoría prestada a las personas ayudando a mejorar su conducta y propiciando una mejor autoeficacia en la lucha por la abstinencia ${ }^{31,32}$.

Por otro lado, se puede mencionar que el tema de intervenciones en enfermería para favorecer la cesación tabáquica, así como muchos otros temas de la salud, demuestran poca evidencia científica producida a nivel de Latinoamérica ${ }^{33}$; los estudios encontrados en esta revisión, corresponden a investigaciones extranjeras, por lo que se genera la necesidad de buscar una mejor actuación en la problemática del tabaquismo, desde diferentes poblaciones y contextos.

\section{REFERENCIAS BIBLIOGRÁFICAS}

${ }^{1}$ World Health Organization. Who report on the global tobacco epidemic, 2015. Raising taxes on tobacco. [Internet]. [Consultado 16 enero 2018]. Disponible en:

http://apps.who.int/iris/bitstream/10665/ 178574/1/9789240694606_eng.pdf?ua= $1 \& u a=1$

2.Figueira-Pereira C, De Vargas D. Profile of women who carried out smoking cessation treatment: a systematic review. Rev Saúde Pública. 2015; 49: 40.

${ }^{3}$ Ministerio de Salud y Protección Social (MINSALUD). Generación más: somos más sin tabaco. Colombia. 2018. [Internet]. [Consultado el 16 de enero del 
Intervenciones no farmacológicas realizadas por...

2018]. Disponible en: https://www.minsalud.gov.co/Paginas/G eneración\%20más\%20\%20somos\%20m ás\%20sin\%20tabaco.aspx

4. República de Colombia. Congreso de la República de Colombia. Ley número 1335 de 2009. Regulación para el consumo, venta, publicidad y promoción del cigarrillo, tabaco y sus derivados. [Internet]. [Consultado el 16 de enero del 2018]. Disponible en: http://media.utp.edu.co/atencionalciudad ano/archivos/Ley\%201335\%20de\%202 009.pdf

5. Pardo C, Piñeros M. Consumo de tabaco en cinco ciudades de Colombia, Encuesta Mundial de Tabaquismo en Jóvenes, 2007. Biomédica 2010; 30: 509-18.

6. Pinilla-Vásquez C, Angarita-Fonseca A. Conocimientos y actitudes asociadas al inicio del hábito de fumar durante la vida universitaria. Hacia la Promoción de la Salud. 2012; 17 (2): 25-39.

7. Cogollo Z, Gómez-Bustamante EM. Asociación entre consumo de cigarrillo, alcohol y sustancias ilegales en adolescentes estudiantes en Cartagena, Colombia, 2012. Hacia la Promoción de la Salud. 2013; 18 (1): 110-17.

${ }^{8}$ Shaheen-Shaik S, Dolar D, ReddyBandari S, Reddy- Madupu P, Kulkarni S. Tobacco Use Cessation and Prevention - A Review. J Clin Diagn Res. 2016; 10(5): ZE13-ZE17.

9. Olano-Espinosa E, Minué-lorenzo C. «No hacer», también en tabaco. Aten Primaria. 2016; 48 (7): 493-99.

10. Alba LH, Murillo RH, Becerra NA, Páez $\mathrm{N}$, Cañas $\mathrm{A}$, Mosquera $\mathrm{CM}$, et al. Recomendaciones para la cesación de la adicción al tabaco en Colombia. Biomédica. 2013; 33: 186-204.
${ }^{11}$ Wu L, He Y, Jiang B, Zuo F, Lui Q, Zhang L, et al. Effectiveness of additional follow-up telephone counseling in a smoking cessation clinic in Beijing and predictors of quitting among Chinese male smokers. Wu et al. BMC Public Health (2016) 16:63.

${ }^{12}$ Momin B, Neri A, McCausland K, Duke J, Hansen H, Kahende J. Traditional and Innovative Promotional Strategies of Tobacco Cessation Services: A Review of the Literature. Journal of Community Health.2014; 39 (4): 800-09.

${ }^{13}$ Ortega- Garcia J, Perales JE, CarecelesAlvarez A, Sanchez-Sauco MF, Villalona S, Mondejar-Lopez $\mathrm{P}$, et al. Seguimiento a largo plazo de un programa de prevención y cesación tabáquica en pacientes con fibrosis quística. Adiciones. 2016; 28 (2): 99107.

${ }^{14}$ Jimenez-Ruiz CA, Solano-Reina S, de Granda-Orive JI, Signes-Costa Minaya J, Martinez EH, Riesco-Miranda JA, et al. El cigarrillo electrónico. Declaración oficial de la Sociedad Espanola de Neumología y Cirugía Torácica (SEPAR) sobre la eficacia, seguridad y regulación de los cigarrillos electrónicos. Arch Bronconeumol. 2014; 50(8): 36267.

${ }^{15}$ Berit-Petersen A, Meyer B, Sachs BL, Bialous SA, Cataldo JK. Preparing nurses to intervene in the tobacco epidemic: Developing a model for faculty development and curriculum redesign. Nurse Education in Practice. 2017; 25: 29-35.

${ }^{16}$.Fore AM, Karvonen-Gutierrez CA, Talsma A, Duffy SA. Nurses' delivery of the Tobacco Tactics intervention at a 
Valencia, C., Palacio, V., Villada, L., Giraldo, K.

Veterans Affairs Medical Center. Journal of Clinical Nursing. 2013; 23, 2162-69.

17. Cato K, Hyun S, Bakken S. Response to a Mobile Health Decision Support System for Screening and Management of Tobacco Use. Oncol Nurs Forum. 2014; 41(2): 145-52.

18. Gálvez-Toro A. Un enfoque crítico para la construcción de una enfermería basada en evidencia Investigación y Educación en Enfermería. 2003; 21(1): 50-63.

${ }^{19}$ Berndt N, Bolman C, Lechner L, Max W, Mudde A, de Vries H, et al. Economic evaluation of a telephone- and face-toface-delivered counseling intervention for smoking cessation in patients with coronary heart disease. Eur J Health Econ. 2016; 17(3):269-85.

${ }^{20}$ Li WH1, Wang MP1, Lam TH2, Cheung YT1, Cheung DY2, Suen YN. Brief intervention to promote smoking cessation and improve glycemic control in smokers with type 2 diabetes: a randomized controlled trial. Sci Rep. 2017; 7:45902.

${ }^{21 .}$ Chan SS, Wong DC, Cheung YT, Leung DY, Lau L, Lai V, et al. A block randomized controlled trial of a brief smoking cessation counselling and advice through shortmessage service on participants who joined the Quit to Win Contest in Hong Kong. Health Educ Res. 2015; 30(4):609-21.

${ }^{22 .}$ Choi SH1, Waltje AH, Ronis DL, Noonan D, Hong O, Richardson CR, et al. Web-enhanced tobacco tactics with telephone support versus 1-800-QUITNOW telephone line intervention for operating engineers: randomized controlled trial. J Med Internet Res. 2014; 16(11):e255.
${ }^{23}$ Kim SS. A Culturally Adapted Smoking Cessation Intervention for Korean Americans: Preliminary Findings. J Transcult Nurs. 2017; 28(1):24-31.

${ }^{24}$ Duffy SA, Ronis DL, Ewing LA, Waltje AH, Hall SV, Thomas PL, et al. Implementation of the Tobacco Tactics intervention versus usual care in Trinity Health community hospitals. Implement Sci. 2016; 11(1):147.

${ }^{25}$ Duffy SA, Ronis DL, KarvonenGutierrez CA, Ewing LA, Hall SV, Yang JJ. Effectiveness of the Tobacco Tactics Program in the Trinity Health System. Am J Prev Med. 2016; 51(4):551-65.

${ }^{26 .}$ Almaraz-Castruita DA, Alonso-Castillo MM, López-García KS, Gómez-Meza MV, Rodríguez-Carvajal R. Intervención breve multicomponente de enfermería para reducir el consumo de tabaco: un estudio de factibilidad. Journal Health NPEPS. 2018; 3(1):67-87.

${ }^{27}$ Rice VH, Heath L, Livingstone-Banks J, Hartmann-Boyce J. Nursing interventions for smoking cessation. Cochrane Database of Systematic Reviews. 2017; 12: CD001188.

28. Almaraz-Castruita DA, Alonso-Castillo MM. Terapia Cognitivo Conductual para Dejar de Fumar: Revisión Sistemática. Enfermería Comunitaria, 2018; 14: e11470.

${ }^{29}$ Blanco-Riopedre $\mathrm{MC}, \quad$ FernándezFernández E. Efectividad de un programa de deshabituación tabáquica llevado a cabo en el centro de salud de Tapia de Casariego (Principado de Asturias). Enfermería Comunitaria. 2015; 3(2):23-34.

30. Olano-Espinosa E, Minué-Lorenzo C. «No hacer», también en Tabaco. Aten 
Intervenciones no farmacológicas realizadas por...

Primaria. 2016;48(7):493-99.

31. Alba LH, Murillo R, Castillo JS. Intervenciones de consejería para la cesación de la adicción al tabaco: revisión sistemática de la literatura. Salud pública de México. 2013; 55 (2): 196-206.

32. Pérez-Milena A, Navarrete-Guillén AB, Mesa-Gallardo MI, Martínez-Pérez R, Leal-Helmling FJ, Pérez-Fuentes C.
Eficiencia de dos intervenciones motivacionales para la deshabituación tabáquica en adolescentes (breve e intensiva) realizadas en Institutos de Educación Secundaria. Adicciones, 2012; 24 (3): 191-200.

${ }^{33}$ Sánchez-Tarragó N, Santiago Bufrem L, Macedo dos Santos RN. La producción científica latinoamericana desde una mirada poscolonial. Tend. Pesqui. Bras. Ciênc. Inf. 2015; 8(2): 182-202. 